\title{
Use of the Mobile Post-Operative Wound Evaluator in the Management of Deep Surgical Site Infection after Abdominal Wall Reconstruction
}

\author{
Ravi F. Sood,' Andrew S. Wright, Heidi Nilsen, JoAnne D. Whitney, \\ William B. Lober, ${ }^{2-4}$ and Heather L. Evans ${ }^{1}$
}

\begin{abstract}
Background: Surgical site infections (SSIs) after ventral hernia repair typically occur after discharge from the hospital, making timely diagnosis difficult. Successful treatment of SSIs may require re-admission and weeks to months of care and follow-up visits, which is potentially costly and burdensome to patients. To address these challenges, we have developed the Mobile Post-Operative Wound Evaluator (mPOWEr), a mobile health application that allows patients to record and transmit SSI symptom data and photographs to clinicians.

Case Presentation: A 45-year-old female underwent open repair of a large recurrent ventral hernia with component separation and synthetic mesh sub-lay. After noting a change in the character of her drain output and transmitting the information via mPOWEr, she was diagnosed with a deep SSI requiring re-admission, operative washout, and negative pressure therapy. This management resulted in mesh salvage and healing without hernia recurrence, documented serially using mPOWEr.

Conclusions: Diagnosis and treatment of SSIs after ventral hernia repair is challenging for physicians and patients alike. Mesh salvage is feasible in deep SSIs involving lightweight macroporous polypropylene mesh, but may require protracted care. Mobile health solutions that allow transmission of patient-generated health data can facilitate early diagnosis of SSI and long-term monitoring of outpatient treatment.
\end{abstract}

Keywords: abdominal wall reconstruction; component separation; mHealth; patient-generated health data; surgical site infection; ventral hernia repair

W ITH MODERN ADVANCES in hernia surgery including component-separation techniques, surgeons are able to repair increasingly large and complex ventral hernias with low recurrence rates [1]. However, post-operative surgical site infections (SSIs) remain common, affecting more than $20 \%$ of open ventral hernia repairs [2]. Treatment of these infections typically requires a combination of antibiotic therapy, wound care, percutaneous drain placement, or reoperation, in some cases with mesh explantation. Thus, it is perhaps not surprising that SSIs have been shown to be the overall costliest healthcare-associated infection [3].
In addition to being costly, SSIs can pose diagnostic challenges, because $60 \%$ of SSIs occur after hospital discharge [4], placing patients at risk for delayed diagnosis and attendant septic complications. Moreover, given that more than half of patients who develop an SSI after discharge are re-admitted to the hospital and many require ongoing SSI care with close incision monitoring, even non-operatively treated SSIs can be burdensome to patients, in particular those who live far from their surgical care environment or have limited mobility or transportation means. In an effort to aid in early diagnosis and treatment of SSIs, we have

\footnotetext{
${ }^{1}$ Department of Surgery, ${ }^{2}$ Department of Biobehavioral Nursing and Health Informatics, School of Nursing, ${ }^{3}$ Department of Global Health, School of Public Health, ${ }^{4}$ Department of Biomedical Informatics and Medical Education, School of Medicine, University of Washington, Seattle, Washington.

(C) Ravi F. Sood et al. 2017; Published by Mary Ann Liebert, Inc. This Open Access article is distributed under the terms of the Creative Commons License (http://creativecommons.org/licenses/by/4.0), which permits unrestricted use, distribution, and reproduction in any medium, provided the original work is properly credited.
} 
developed the Mobile Post-Operative Wound Evaluator (mPOWEr), a patient-centered mobile health (mHealth) application accessible via smartphone, tablet, or computer that combines a validated post-discharge SSI surveillance questionnaire with patient-generated incision photographs [5]. In this report, we describe a case of deep SSI after abdominal wall reconstruction that was treated successfully with salvage of the involved synthetic mesh, highlighting the utility of mHealth-transmitted patient-generated health data in SSI management.

\section{Case Presentation}

A 45-year-old female presented to our clinic with an 8-year history of progressive abdominal bulge associated with debilitating pain. She had undergone a mesh repair of a primary umbilical hernia 13 years prior. On physical examination, she had a large irreducible ventral hernia (Fig. 1A). Computed tomography (CT) corroborated the physical examination findings, demonstrating an $11 \mathrm{~cm}$ (craniocaudad) by $8 \mathrm{~cm}$ (transverse) fascial defect with loss of abdominal domain (Fig. 1B-1C); an associated large hernia sac contained most of the small and large bowel, including an inflamedappearing loop of ileum suspicious for entero-enteric fistula (Fig. 1B). Additional medical history included morbid obesity (weight, $135 \mathrm{~kg}$; body mass index [BMI] 51 at her initial clinic visit), type 2 diabetes mellitus, and active smoking. Over the ensuing 15 months, she lost $36 \mathrm{~kg}$ in a medically supervised weight-loss program (reducing her BMI to 37), improved her blood glucose control, and achieved complete smoking cessation. Having optimized her pre-operative risk factors, we planned a retro-rectus mesh repair with an option to perform bilateral transversus abdominus release to restore abdominal domain and recreate the mid-line with wide mesh reinforcement.

We began her operation with a mid-line laparotomy and extensive lysis of adhesions. Approximately $50 \mathrm{~cm}$ of hardened, abnormal ileum was densely adherent to the abdominal wall, consistent with a contained perforation and chronic entero-enteric fistula. We dissected this segment free, resected it, and performed a stapled anastomosis. There was no gross spillage of enteric contents. Pathologic review of this specimen revealed mesh from the patient's prior umbilical hernia repair within the small bowel lumen. After completing adhesiolysis, we noted that the fascial edges were more than $10 \mathrm{~cm}$ apart under great tension (Fig. 2A). This necessitated creation of bilateral myofascial advancement flaps, extending our dissection into the retro-rectus space to accommodate a bilateral transversus abdominus release [1]. We closed the posterior rectus sheath (Fig. $2 \mathrm{~B}$ ), placed a $60 \times 60 \mathrm{~cm}$ macroporous polypropylene mesh sub-lay in the retro-rectus plane (Fig. 2C), and then closed the anterior rectus sheath. We placed closed-suction drains in both the retro-rectus and subcutaneous spaces and closed the subcutaneous tissue in layers after excising the hernia sac and $300 \mathrm{~cm}^{2}$ of ischemic skin, long attenuated by the hernia contents (Fig. 2D). Operative time (incision to closure) was 9 hours and 25 minutes.

The patient's initial post-operative course was unremarkable, and she was discharged from the hospital on the third post-operative day with drains in place. On postoperative day 6 , the patient noted a change in character of the fluid in her subcutaneous drain from light and clear to dark, cloudy, and malodorous, as she documented in mPOWEr's "Drain Tracking" feature. As a result, she was seen in clinic the following day and given a prescription for antibiotic agents given the purulent appearance of her drain output (Fig. 3A). Whereas she initially was clinically well, she subsequently began reporting chills and malaise and thus was re-admitted on post-operative day 12 and started on intravenous antibiotic agents. Computed tomography demonstrated a subcutaneous fluid collection consistent with deep SSI. We returned to the operating room for incision and drainage, evacuating approximately $100 \mathrm{~mL}$ of frank purulence and debriding an area of non-viable anterior rectus sheath, leaving a $15 \mathrm{~cm}$ length of intact exposed mesh (Fig. 3B). We initiated negative-pressure wound therapy with instillation of dilute Dakin solution until the first dressing change at 48 hours; by the second vac change on post-operative day 16 , there was
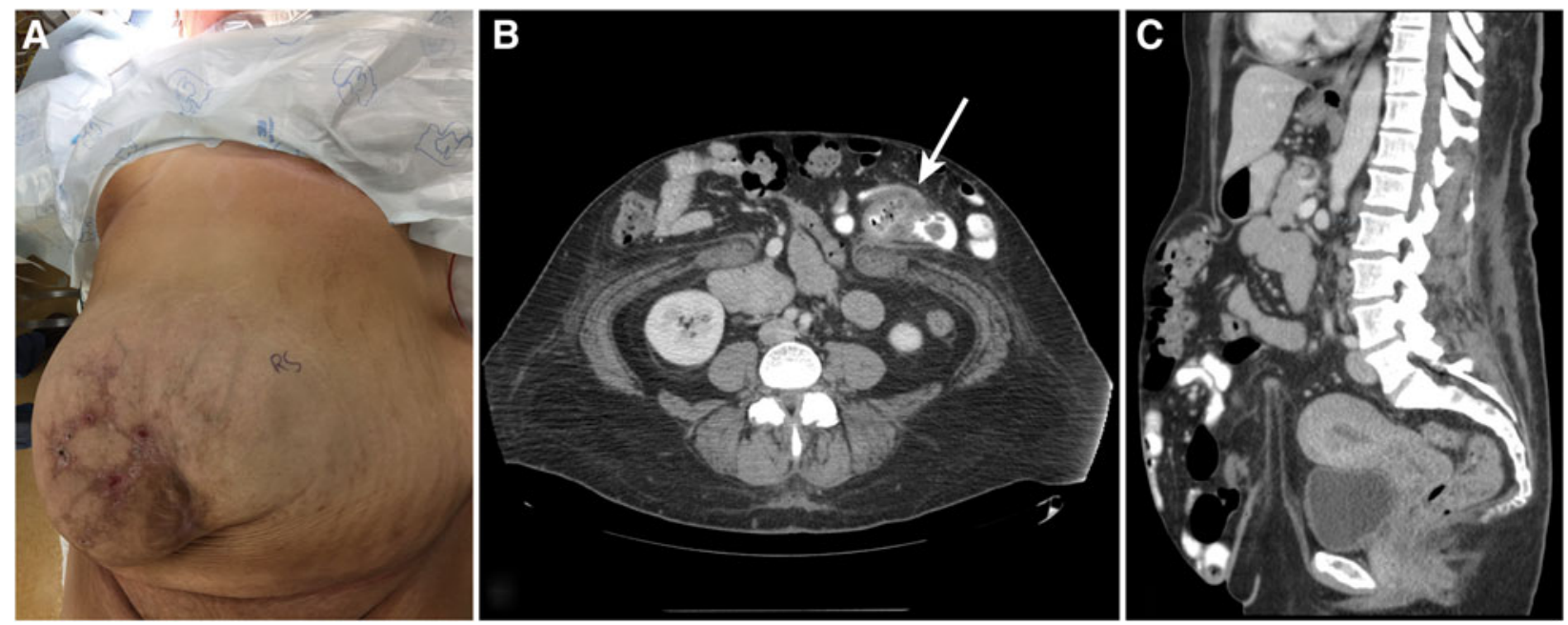

FIG. 1. Pre-operative hernia assessment. (A) Pre-operative photograph showing ulcerated skin overlying large hernia sac.

(B) Axial cut from pre-operative computed tomography (CT) scan; white arrow indicates inflamed loop of ileum. (C) Sagittal cut from pre-operative CT scan demonstrating large hernia sac with loss of intra-abdominal domain. 

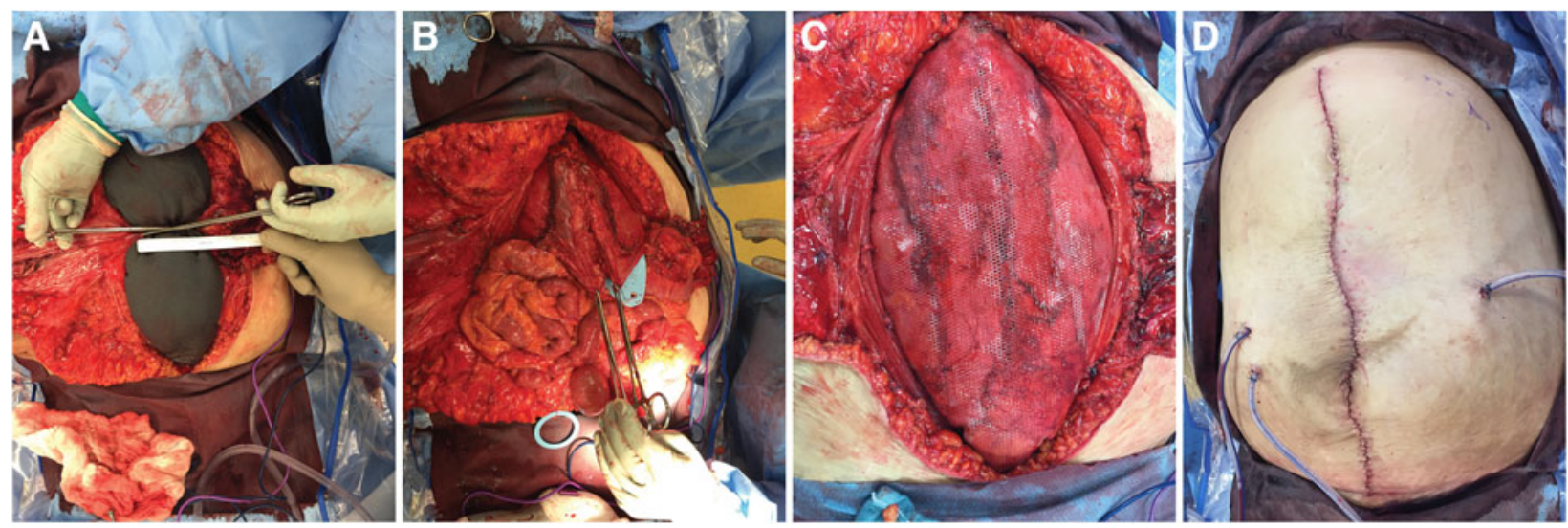

FIG. 2. Intra-operative photographs. (A) Fascial defect prior to posterior component release. (B) Partial closure of posterior rectus sheath following bilateral transversus abdominus release (TAR). (C) Polypropylene mesh sub-lay placed over closed posterior rectus sheath. (D) Immediate post-operative appearance of the abdomen after drain placement and skin closure.

robust granulation tissue and no evidence of ongoing infection (Fig. 3C). The operative cultures grew multi-drug-resistant Proteus mirabilis, Klebsiella pneumoniae, and Enterobacter cloacae. The patient was discharged home on post-operative day 21 , where she continued negative-pressure wound therapy and completed a two-week course of intravenous ertapenem. She continued to recover well clinically and her incision healed completely by approximately three months post-operative, as documented with serial photos in $\mathrm{mPOWEr}$ (Fig. 4). The patient is without recurrence or infection at nine months post-operative.

\section{Discussion}

Mesh infection after ventral hernia repair can be a devastating complication, because historically it has typically re- quired re-operation for mesh removal, leading to hernia recurrence and the need for additional operations. Accordingly, careful pre-operative assessment and optimization of SSI risk factors is of paramount importance in minimizing occurrence of mesh infection. At her initial presentation, our patient had three modifiable risk factors for SSI: morbid obesity, diabetes mellitus, and smoking [6]. Pre-operatively, she was able to achieve considerable weight loss, excellent blood glucose control, and complete smoking cessation, favorably altering her SSI risk profile. However, she had several other SSI risk factors that were not modifiable: American Society of Anesthesiologists (ASA) score of 3, concomitant bowel resection, and relatively long operative time [6,7] because of the complexity of her case. These factors may have contributed to her development of a deep-space (i.e., mesh) infection.
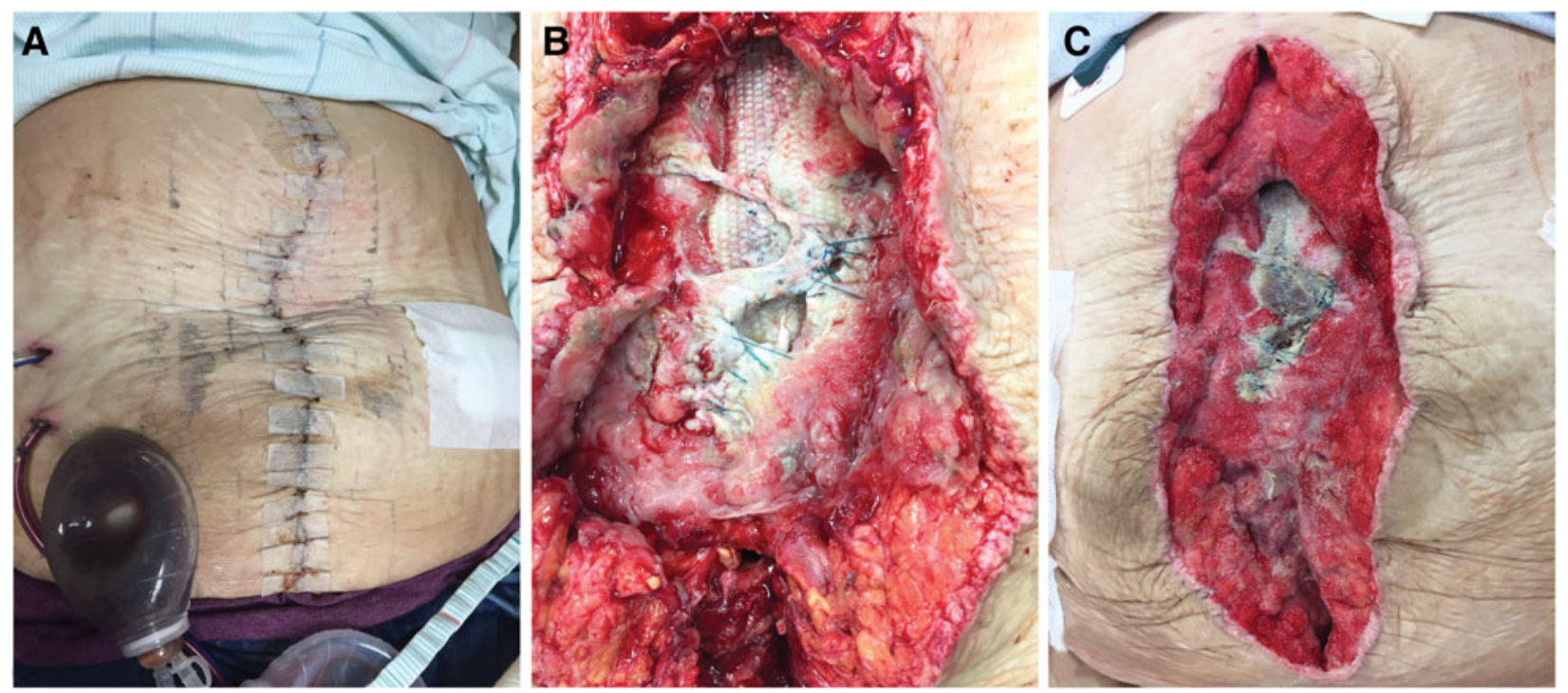

FIG. 3. Early post-operative photographs. (A) Dark purulent output in the subcutaneous drain at post-operative day 7. (B) Initial appearance at post-operative day 12 operative washout, edges of partially debrided anterior rectus sheath, and underlying exposed polypropylene mesh. (C) Incision appearance at post-operative day 16, demonstrating healthy granulation tissue without purulence. 

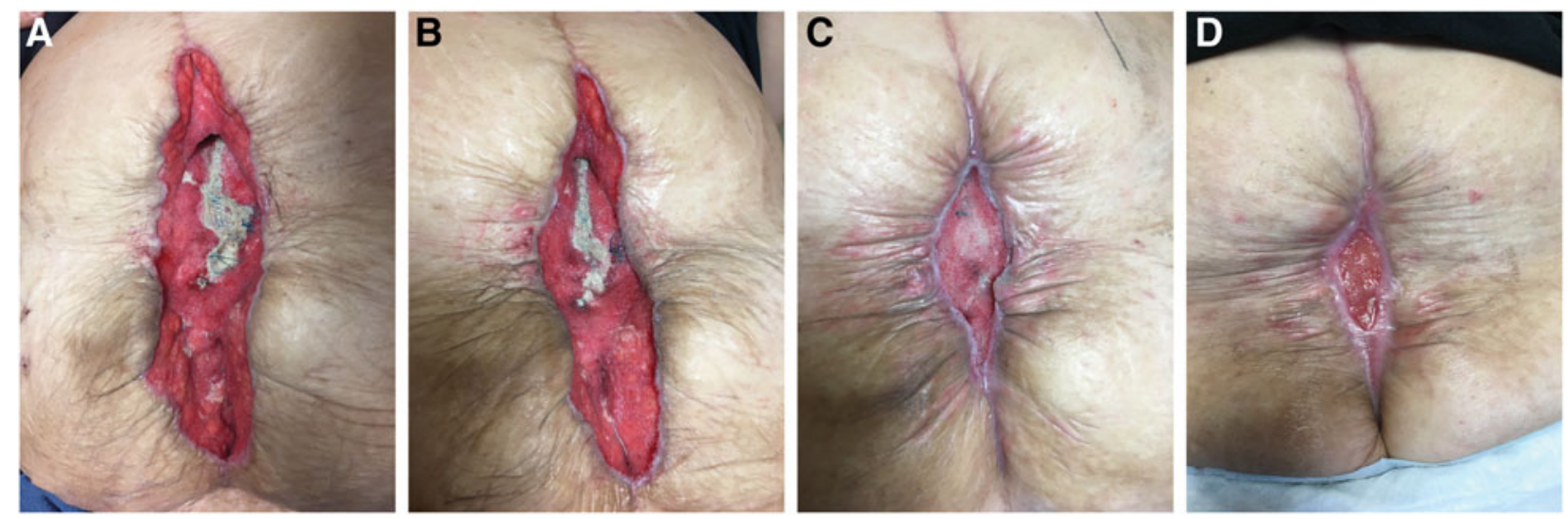

FIG. 4. Late post-operative photographs. Progression of healing incision with negative pressure therapy at post-operative day $26(\mathbf{A}), 33(\mathbf{B}), 63(\mathbf{C})$, and 91 (D).

Given the presence of these non-modifiable risk factors for SSI, in particular the unavoidable contamination introduced by the small bowel resection for her chronic entero-enteric fistula related to prior hernia mesh, our decision to use permanent, synthetic mesh in our repair warrants comment. Until recently, standard practice in the United States has been to avoid synthetic mesh in contaminated fields, instead favoring biologic mesh. The rationale for this practice had been fear of mesh infection requiring mesh explanation. However, there are no high-quality data to support this practice, and with the advent of modern lightweight macroporous monofilament polypropylene mesh-shown to be much more resistant to infection, likely because of decreased surface area for bacterial adherance-there has been increased enthusiasm for using synthetic mesh in contaminated fields [8]. Multiple recent reports confirm that although ventral hernia repair in clean-contaminated and contaminated fields are unsurprisingly associated with high incidence of SSIs, the vast majority of these infections can be treated successfully with negative pressure therapy, avoiding the need for mesh explanation often required in the prior era of dense, microporous, multifilament mesh $[9,10]$. Our case report adds to this growing literature supporting the practice of using synthetic mesh in contaminated hernia repair, because despite the occurrence of a deep SSI, our patient has made a full recovery with mesh salvage and has no sign of hernia recurrence at nine months post-operative.

This case also illustrates the utility of mHealth-transmitted patient-generated health data in diagnosing and treating SSI. Our patient documented and communicated the change in the character of her drain output via the mPOWEr application, ultimately leading to timely re-admission and initiation of treatment and therefore potentially helping to avoid sepsis or other serious sequelae. In addition, we and the patient used mPOWEr to document serially the progress of her wound, both while she was an inpatient and then during months of outpatient incision care. The patient and providers believe that patient-generated incision photographs enhanced communication and engendered confidence in the care delivered. This was especially important because the patient lived more than an hour from the hospital. The secure transmission and storage of incision photographs allowed providers to track the progress of the incision accurately over time with fewer clinic visits, mitigating some of the burden associated with SSI. Until recently, the use of $\mathrm{mPOWEr}$ has been limited to the University of Washington, but we are currently in the process of expanding its availability and studying its clinical efficacy in hopes it may ultimately be used widely to improve outcomes related to SSIs.

\section{Conclusions}

Diagnosis and treatment of SSIs after ventral hernia repair can be challenging for physicians and patients alike. Mesh salvage is feasible in deep SSIs involving lightweight macroporous monofilament polypropylene mesh, supporting the use of synthetic mesh in contaminated hernia repairs. Mobile health solutions such as mPOWEr may facilitate early diagnosis and long-term management of SSIs.

\section{Author Disclosure Statement}

The authors have no competing financial interests.

\section{References}

1. Pauli EM, Rosen MJ. Open ventral hernia repair with component separation. Surg Clin North Am 2013;93:1111-1133.

2. Berger RL, Li LT, Hicks SC, et al. Development and validation of a risk-stratification score for surgical site occurrence and surgical site infection after open ventral hernia repair. J Am Coll Surg 2013;217:974-982.

3. Sanger PC, Hartzler A, Han SM, et al. Patient perspectives on post-discharge surgical site infections: Towards a patientcentered mobile health solution. PLoS One 2014;9:e114016.

4. Woelber E, Schrick EJ, Gessner BD, et al. Proportion of surgical site infections occurring after hospital discharge: A systematic review. Surg Infect 2016;17:510-519.

5. Evans HL, Lober WB. A pilot use of patient-generated wound data to improve postdischarge surgical site infection monitoring. JAMA Surg 2017;152:595-596.

6. Mavros MN, Athanasiou S, Alexiou VG, et al. Risk factors for mesh-related infections after hernia repair surgery: A meta-analysis of cohort studies. World J Surg 2011;35: 2389-2398.

7. Finan KR, Vick CC, Kiefe CI, et al. Predictors of wound infection in ventral hernia repair. Am J Surg 2005;190:676-681. 
8. Carbonell AM, Cobb WS. Safety of prosthetic mesh hernia repair in contaminated fields. Surg Clin North Am 2013;93: 1227-1239.

9. Carbonell AM, Criss CN, Cobb WS, et al. Outcomes of synthetic mesh in contaminated ventral hernia repairs. J Am Coll Surg 2013;217:991-998.

10. Meagher H, Clarke Moloney M, Grace PA. Conservative management of mesh-site infection in hernia repair surgery: A case series. Hernia 2015;19:231-237.

Address correspondence to:

Dr. Ravi F. Sood

University of Washington 1959 NE Pacific Street

Seattle, WA 98195

E-mail:rfsood@uw.edu

\begin{aligned} & \hline Abbreviations Used \\ & $\mathrm{BMI}=$ body mass index \\ & $\mathrm{CT}=$ computed tomorgraphy \\ & $\mathrm{mHEALTH}=$ mobile health \\ & $\mathrm{mPOWEr}=$ Mobile Post-Operative Wound \\ & $\mathrm{SSI}=$ Evargical site infection \\ & $\mathrm{TAR}=$ transversus abdominus release \\ &\end{aligned}

Cite this article as: Sood RF, Wright AS, Nilsen $\mathrm{H}$, Whitney JD, Lober WB, Evans HL. (2017) Use of the Mobile Post-Operative Wound Evaluator (mPOWEr) in the management of deep surgical site infection after abdominal wall reconstruction. Surgical Infections Case Reports 2:1, 80-84, DOI: 10.1089/crsi.2017.0022 